\title{
Contralateral $\mathrm{C} 7$ to $\mathrm{C} 7$ nerve root transfer in reconstruction for treatment of total brachial plexus palsy: anatomical basis and preliminary clinical results
}

\author{
${ }^{*}$ Guo-Bao Wang, MM, ${ }^{1,2}$ Ai-Ping Yu, MB, $, 1,2$ Chye Yew Ng, FRCS, ${ }^{3}$ Gao-Wei Lei, MB, ${ }^{1}$ \\ Xiao-Min Wang, MB, ${ }^{2}$ Yan-Qun Qiu, MD, ${ }^{2}$ Jun-Tao Feng, MD, ${ }^{1}$ Tie Li, MD, ${ }^{1}$ Qing-Zhong Chen, MD, ${ }^{4}$ \\ Qian-Ru He, PhD, ${ }^{5}$ Fei Ding, PhD, ${ }^{5}$ Shu-Sen Cui, MD, PhD, ${ }^{6}$ Yu-Dong Gu, MD, PhD, ${ }^{1}$ \\ Jian-Guang $\mathrm{Xu}, \mathrm{MD}, \mathrm{PhD},{ }^{1}$ Su Jiang, MD, ${ }^{1}$ and Wen-Dong $\mathrm{Xu}, \mathrm{MD}, \mathrm{PhD}^{1,2,5,7-9}$
}

\begin{abstract}
'Department of Hand Surgery, Huashan Hospital, Shanghai Medical College, Fudan University, Shanghai, China; ${ }^{2}$ Department of Hand and Upper Extremity Surgery, Jing'an District Central Hospital, Shanghai, China; ${ }^{3}$ ppper Limb Unit, Wrightington Hospital, Wigan, United Kingdom; ' Department of Hand Surgery, Affiliated Hospital of Nantong University, Nantong, Jiangsu Province, China; ${ }^{5}$ Key Laboratory of Neuroregeneration of Jiangsu and Ministry of Education, Co-Innovation Center of Neuroregeneration, Nantong University, Nantong, Jiangsu Province, China; ${ }^{6}$ Department of Hand Surgery, China-Japan Union Hospital of Jilin University, Changchun, Jilin Province, China; ${ }^{7}$ State Key Laboratory of Medical Neurobiology, Collaborative Innovation Center of Brain Science, Fudan University, Shanghai, China; ${ }^{8}$ Priority Among Priorities of Shanghai Municipal Clinical Medicine Center, Shanghai, China; and ${ }^{9}$ National Clinical Research Center for Aging and Medicine, Huashan Hospital, Fudan University, Shanghai, China
\end{abstract}

OBJECTIVE Contralateral C7 (CC7) nerve root has been used as a donor nerve for targeted neurotization in the treatment of total brachial plexus palsy (TBPP). The authors aimed to study the contribution of $\mathrm{C} 7$ to the innervation of specific upper-limb muscles and to explore the utility of $\mathrm{C} 7$ nerve root as a recipient nerve in the management of TBPP.

METHODS This was a 2-part investigation. 1) Anatomical study: the $C 7$ nerve root was dissected and its individual branches were traced to the muscles in 5 embalmed adult cadavers bilaterally. 2) Clinical series: 6 patients with TBPP underwent CC7 nerve transfer to the middle trunk of the injured side. Outcomes were evaluated with the modified Medical Research Council scale and electromyography studies.

RESULTS In the anatomical study there were consistent and predominantly C7-derived nerve fibers in the lateral pectoral, thoracodorsal, and radial nerves. There was a minor contribution from $\mathrm{C} 7$ to the long thoracic nerve. The average distance from the $\mathrm{C} 7$ nerve root to the lateral pectoral nerve entry point of the pectoralis major was the shortest, at 10.3 $\pm 1.4 \mathrm{~cm}$. In the clinical series the patients had been followed for a mean time of $30.8 \pm 5.3$ months postoperatively. At the latest follow-up, 5 of 6 patients regained M3 or higher power for shoulder adduction and elbow extension. Two patients regained M3 wrist extension. All regained some wrist and finger extension, but muscle strength was poor. Compound muscle action potentials were recorded from the pectoralis major at a mean follow-up of $6.7 \pm 0.8$ months; from the latissimus dorsi at $9.3 \pm 1.4$ months; from the triceps at $11.5 \pm 1.4$ months; from the wrist extensors at $17.2 \pm 1.5$ months; from the flexor carpi radialis at $17.0 \pm 1.1$ months; and from the digital extensors at $22.8 \pm 2.0$ months. The average sensory recovery of the index finger was $\mathbf{2} 2$. Transient paresthesia in the hand on the donor side, which resolved within 6 months postoperatively, was reported by all patients.

CONCLUSIONS The $\mathrm{C} 7$ nerve root contributes consistently to the lateral pectoral nerve, the thoracodorsal nerve, and long head of the triceps branch of the radial nerve. CC7 to C7 nerve transfer is a reconstructive option in the overall

ABBREVIATIONS AN = axillary nerve; $\mathrm{BMRC}=$ British Medical Research Council; $\mathrm{CC} 7=$ contralateral $\mathrm{C7} ; \mathrm{EMG}=$ electromyography; $\mathrm{FCR}=$ flexor carpi radialis; ICN = intercostal nerve; $\mathrm{LHTB}=$ long head of the triceps branch; $\mathrm{LPN}=$ lateral pectoral nerve; $\mathrm{LTN}=$ long thoracic nerve; $\mathrm{PM}=$ pectoralis major; $\mathrm{PN}=$ phrenic nerve; $\mathrm{SAN}=\mathrm{spi}-$ nal accessory nerve; SSN = suprascapular nerve; TBPP = total brachial plexus palsy; TN = thoracodorsal nerve; 2 -PD = 2-point discrimination.

SUBMITTED November 15, 2017. ACCEPTED March 14, 2018.

INCLUDE WHEN CITING Published online August 3, 2018; DOI: 10.3171/2018.3.SPINE171251.

* G.B.W. and A.P.Y. contributed equally to this work. S.J. and W.D.X. contributed equally to this work and share senior authorship. 
management plan for TBPP. It was safe and effective in restoring shoulder adduction and elbow extension in this patient series. However, recoveries of wrist and finger extensions are poor.

https://thejns.org/doi/abs/10.3171/2018.3.SPINE171251

KEYWORDS contralateral C7; total brachial plexus palsy; nerve transfer; donor nerve

I N 1992, Gu et al. ${ }^{11}$ reported the concept and clinical application of using a healthy contralateral C7 (CC7) nerve root as donor nerve in reconstruction of the brachial plexus for treatment of total brachial plexus palsy (TBPP). Since then CC7 nerve transfer has gained wider acceptability, with promising results reported from other centers. ${ }^{6,26,28,29,31-34}$ Apart from TBPP, the utility of CC7 has also been expanded to the treatment of patients with central neurological injuries, such as cerebral palsy, traumatic brain injury, and stroke. ${ }^{14,37,39}$

The procedure's premise is that the harvest of $\mathrm{C} 7$ nerve root would not cause permanent functional loss in the healthy extremity. Following transection of the $\mathrm{C} 7$ nerve root, voluntary control over the muscles that it innervates appears to be preserved due to compensation by the adjacent nerve roots. For instance, apart from C7 innervation, the latissimus dorsi is also innervated by $\mathrm{C} 6$ and $\mathrm{C} 8$ roots; the triceps by $\mathrm{C} 5, \mathrm{C} 6, \mathrm{C} 8$, and $\mathrm{T} 1$; and the forearm extensors by $\mathrm{C} 6$ and $\mathrm{C} 8$. Previous studies in rodents and humans have shown that the $\mathrm{C} 7$ myotome contributes to the movements of the shoulder, elbow, wrist, and hand. ${ }^{9}, 17$ This is supported by the comprehensive functional improvement observed in the injured upper limb following CC7 to C7 transfer in patients with central neurological injury. ${ }^{14}$ Some anatomical reports have revealed the structure of C7 and brachial plexus by cadaveric, ${ }^{12,25}$ histochemical, $, 6,35$ or electrophysiological studies. ${ }^{9,18}$ However, the relative contribution of $\mathrm{C} 7$ innervation to the overall function of the upper limb remains unknown, as well as the recovery assessment of specific muscles.

In this study, cadaveric and clinical investigations were combined to delineate the neuroanatomy of $\mathrm{C} 7$ nerve fibers innervating the specific upper-limb muscles and to ascertain the extent of function(s) that can be regained by successful reanimation of the $\mathrm{C} 7$ myotome through $\mathrm{CC} 7$ nerve transfer in a completely paralyzed limb.

\section{Methods}

\section{Anatomical Study}

The brachial plexus specimens of 5 embalmed adult cadavers were bilaterally dissected in the Department of Anatomy, Histology, and Embryology at the Medical College of Fudan University. Dissection was performed using loupe magnification $\times 2.5$. Particular attention was paid to the $\mathrm{C} 7$ nerve root, where the distal branches were dissected and followed until they reached a known muscle. The distances from the $\mathrm{C} 7$ root (transverse foramen) to the nerve entry point of target muscles were measured by a ruler.

\section{Clinical Series}

Patient Population

Between November 2012 and January 2014, 8 patients with TBPP underwent brachial plexus exploration and reconstruction. The preliminary diagnosis of TBPP was based on history, physical examination, and electromyography (EMG) and MRI findings. The final diagnosis of total root avulsion was confirmed during surgical exploration. All procedures were performed by the senior author (W.D.X.). All patients underwent CC7 to C7 nerve transfer, in addition to other nerve transfers, as part of the overall reconstructive plan. Of the 8 patients, 1 was lost to follow-up and 1 was excluded because of severe concomitant soft-tissue damage of the arm. Among the remaining 6 patients, 5 were male and 1 was female, and the mean age was $27.2 \pm 9.0$ years (range $18-43$ years). The average interval from injury to operation was $2 \pm 0.6$ months (range 1-3 months) (Table 1).

\section{Surgical Technique of CC7 to C7 Transfer}

The patient was placed in the supine position with a sandbag under the interscapular region. The injured brachial plexus was exposed through a supraclavicular trans-

TABLE 1. General information for 6 patients with TBPP in case series

\begin{tabular}{|c|c|c|c|c|c|c|c|c|c|}
\hline $\begin{array}{l}\text { Yr of } \\
\text { Birth }\end{array}$ & Sex & Handedness & $\begin{array}{c}\text { Age at } \\
\text { Injury (yrs) }\end{array}$ & Cause & Past History & $\begin{array}{l}\text { Affected } \\
\text { Side }\end{array}$ & $\begin{array}{l}\text { Time From Injury } \\
\text { to Op (mos) }\end{array}$ & Intraop Diagnosis & $\begin{array}{c}\text { Duration of } \\
\text { FU (mos) }\end{array}$ \\
\hline 1990 & $M$ & Rt & 23 & Traffic accident & - & $\mathrm{Lt}$ & 1 & Lt TBPP & 27 \\
\hline 1989 & $M$ & $\mathrm{Rt}$ & 24 & Traffic accident & - & $\mathrm{Lt}$ & 2 & Lt TBPP & 34 \\
\hline 1969 & M & Rt & 43 & $\begin{array}{l}\text { Fall damage, mul- } \\
\text { tiple injuries }\end{array}$ & $\begin{array}{l}\text { Smoking, hyper- } \\
\text { glycemia }\end{array}$ & $\mathrm{Lt}$ & 2 & $\begin{array}{l}\text { Lt TBPP, It scapula fracture, } \\
\text { C4-5 disc resection }\end{array}$ & 40 \\
\hline 1995 & $F$ & $\mathrm{Rt}$ & 18 & $\begin{array}{l}\text { Traffic accident, } \\
\text { multiple injuries }\end{array}$ & - & $\mathrm{Lt}$ & 2 & Lt TBPP & 30 \\
\hline 1982 & M & Rt & 32 & Traffic accident & - & $\mathrm{Rt}$ & 3 & Rt TBPP & 28 \\
\hline 1990 & $M$ & $\mathrm{Rt}$ & 23 & Traffic accident & - & $\mathrm{Lt}$ & 2 & Lt TBPP & 26 \\
\hline
\end{tabular}

$\mathrm{FU}=$ follow-up. 


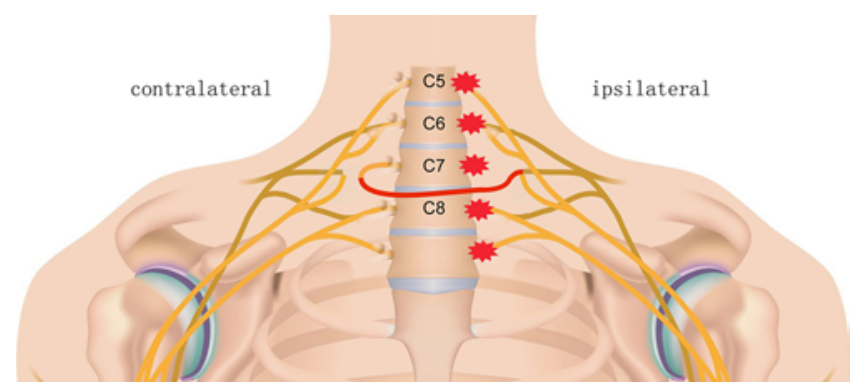

FIG. 1. Schematic drawing of the CC7 transfer procedure. Proximally, the scalenus anterior muscle was severed while protecting the PN. The CC7 nerve root was dissected up to the neural foramen. Distally, the nerve was dissected to the middle trunk as far as its anterior and posterior divisions, and it was transected at the distal end to the divisions. In the retrosternocleidomastoid muscle route, 3-4 strands of the autologous sural nerve grafts were used to bridge the gap between the recipient $\mathrm{C} 7$ nerve root and the donor anterior and posterior divisions of middle trunk. Copyright Su Jiang. Published with permission. Figure is available in color online only.

verse incision. Once the TBPP was confirmed, the target recipient nerves of $\mathrm{C} 7$ nerve root (i.e., the suprascapular nerve [SSN], musculocutaneous nerve, and axillary nerve [AN]) were isolated.

On the uninjured side, a similar supraclavicular incision was made. The CC7 nerve root was isolated and confirmed by intraoperative EMG. During the harvest of the $\mathrm{CC} 7$ root, the scalenus anterior muscle was divided while protecting the phrenic nerve (PN). To obtain maximal nerve length, the nerve root was mobilized up to the neural foramen. The $\mathrm{CC} 7$ nerve was then dissected as distally as its anterior and posterior divisions. The CC7 was transected at the distal end to the divisions after performing lidocaine blockade (1\%, Squibb). The length of the donor nerve was measured.

The sternocleidomastoid muscles on both sides were retracted anteriorly and a tunnel was created by blunt dissection in front of the trachea. Three to four strands of the autologous sural nerve grafts were used to bridge the gap between the recipient and donor $\mathrm{C} 7$ nerve root through the retrosternocleidomastoid route, behind the posterior border of the sternocleidomastoid and in front of the trachea and common carotid artery sheath. Tension-free neurorrhaphy was achieved with 8-0 Ethicon PROLENE (Johnson \& Johnson) under an operating microscope (Fig. 1). After the surgery, a prefabricated brace was used to hold the head in the neutral position, and the injured upper limb was immobilized for a period of 4 weeks.
Rehabilitation commenced at 4 weeks after the surgery. The patients were educated to perform shoulder adduction on the healthy side while undergoing passive exercises of shoulder adduction, elbow extension, and wrist/finger extension on the injured side. Five hundred cycles of exercises per day were recommended. Percutaneous electrical stimulation of the healthy $\mathrm{C} 7$ nerve root was used for at least 6 months postoperatively.

\section{Concurrent Nerve Transfers}

In all patients, the spinal accessory nerve (SAN) was transferred to the SSN, and the motor branches of 4 intercostal nerves (ICNs), III-VI, were transferred to the musculocutaneous nerve in different stages. In 3 patients, the PN was also harvested in full length via video-assisted thoracic surgery and transferred to the anterior branch of the AN.

\section{Evaluation}

All patients were reviewed clinically and underwent EMG testing every 3 months in the first postoperative year. After the first year, they were reviewed every 6 months. Muscle strength was assessed according to the modified British Medical Research Council (BMRC) scale. The grades $\mathrm{M} 0$ to $\mathrm{M} 2$ were regarded as poor, $\mathrm{M} 2+$ to $\mathrm{M} 3$ were fair, M3+ to M4- were good, and M4 to M5- were excellent. Any sensory or motor deficit on the contralateral side was documented.

\section{Statistical Methods}

Statistical analysis was performed with SPSS 15.0 statistical software (SPSS). Data were presented as the mean $\pm \mathrm{SD}$ (range).

\section{Results}

\section{Anatomical Study}

In the cadaver study, there were consistent contributions of C7 nerve root to the lateral pectoral nerve (LPN), thoracodorsal nerve (TN), long thoracic nerve (LTN), and radial nerve, especially the long head of the triceps branch (LHTB) (Table 2, Fig. 2). The distances were recorded from the $\mathrm{C} 7$ root (transverse foramen) to the nerve entry point of target muscles. The supraclavicular and infraclavicular brachial plexus specimens were thoroughly explored and their branches were dissected and traced under loupe magnification $\times 2.5$, whereas distal to the elbow, the attribution of individual nerve fibers from $\mathrm{C} 7$ became less reliable due to the repeated divisions and anastomoses of the fascicles.

TABLE 2. Cadaver study of $\mathrm{C} 7$ root trajectory and its detailed branches

\begin{tabular}{llll}
\hline Nerve & \multicolumn{1}{c}{$\begin{array}{c}\text { Muscle Innervated } \\
\text { (C7 component) }\end{array}$} & C7 Component & $\begin{array}{c}\text { Distance From C7 Nerve Root to Nerve } \\
\text { Entry Point of the Muscle (cm, mean } \pm \text { SD) }\end{array}$ \\
\hline LPN & Clavicular part of the PM & Yes, \& C7 source accounted for the main component & $10.3 \pm 1.38$ \\
\hline TN & Latissimus dorsi & Yes, \& C7 source accounted for the main component & $21.7 \pm 1.77$ \\
\hline LTN & Lower part of the serratus anterior & $\begin{array}{l}\text { Yes, C7 source accounted for minor component, \& the } \\
\text { nerve entry point of the muscle is below the 7th rib level }\end{array}$ & $24.1 \pm 3.26$ \\
\hline LHTB & Long head of the triceps brachii & Yes & $23.4 \pm 1.82$ \\
\hline
\end{tabular}



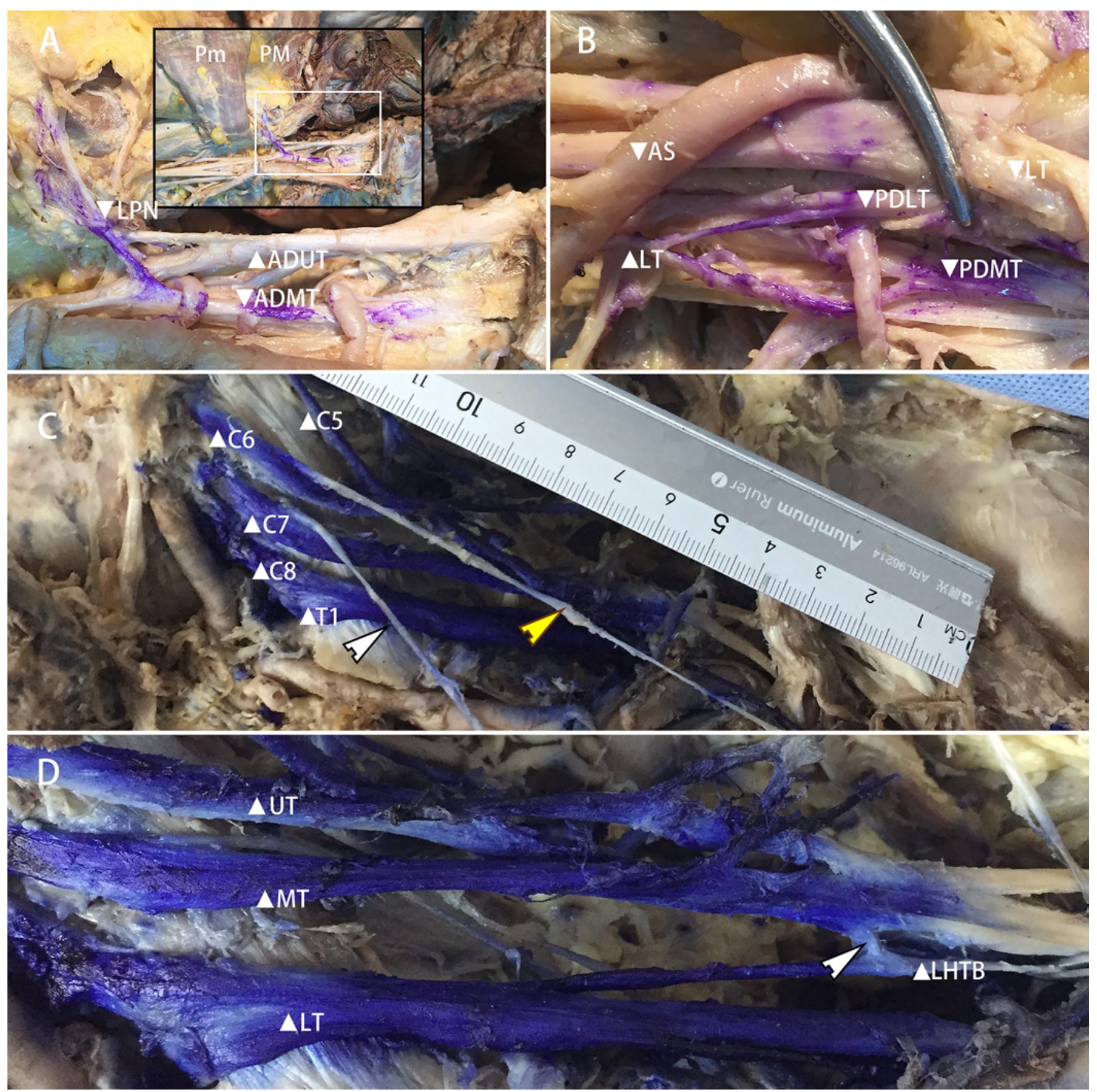

FIG. 2. A: The LPN in this case arose from the anterior divisions of the upper and middle trunk, across the axillary artery and vein, entering the clavicular part of the PM. The LPN and medial pectoral nerve were connected immediately distal to the thoracoacromial artery. B: In this case, the LPN arose from the posterior division of the middle trunk and lower trunk, in which the branch from the middle trunk was thicker in diameter. C: In this case, the LTN came from C6 and C7, the C7 source component accounted for the minor proportion, and the nerve entry point to the muscle is below the level of the seventh ribs. The yellow arrow indicates the nerve component from C6 and the white arrow indicates the nerve component from C7. D: In this case, the LHTB came from the middle and lower trunk. The white arrow indicates the LHTB. ADMT = anterior division of the middle trunk; ADUT = anterior division of the upper trunk; $A S$ = arteria subscapularis; $L T=$ lower trunk; $M T=$ middle trunk; PDLT = posterior division of the lower trunk; PDMT = posterior division of the middle trunk; $\mathrm{Pm}=$ pectoralis minor muscle; $\mathrm{UT}=$ upper trunk. Figure is available in color online only.

\section{Clinical Series}

All patients in our study were confirmed to have TBPP during surgical exploration and underwent $\mathrm{CC} 7$ to $\mathrm{C} 7$ nerve transfer by a single senior surgeon. In the procedure, the average length of dissected CC7 nerve root was $6.5 \pm$ $0.7 \mathrm{~cm}$ (range $4.5-8 \mathrm{~cm}$ ). The mean length of sural nerve 
TABLE 3. Nascent EMG potentials in targeted muscles (months postoperatively) in 6 patients with TBPP

\begin{tabular}{lcccccc}
\hline Case No. & Pectoralis Major & Latissimus Dorsi & Triceps Brachii & Extensor Carpi & Flexor Carpi & Extensor Digitorum \& Pollicis \\
\hline 1 & 7 & 9 & 11 & 17 & 16 & 23 \\
\hline 2 & 6 & 9 & 11 & 17 & 16 & 22 \\
\hline 3 & 8 & 12 & 14 & 20 & 19 & 26 \\
\hline 4 & 6 & 8 & 10 & 17 & 17 & 24 \\
\hline 5 & 7 & 9 & 12 & 16 & 17 & 20 \\
\hline 6 & 6 & 9 & 11 & 16 & 17 & 22 \\
\hline Mean & 6.67 & 9.33 & 11.50 & 17.16 & 17.00 & 22.83 \\
\hline SD & 0.82 & 1.36 & 1.38 & 1.47 & 1.10 & 2.04 \\
\hline
\end{tabular}

graft was $9.5 \pm 2.5 \mathrm{~cm}$ (range $7.5-11.5 \mathrm{~cm}$ ). There were no major intraoperative complications. The mean length of postoperative follow-up was $30.8 \pm 5.3$ months (range 26-40 months). Three patients had concurrent surgery that attached full-length PN to the anterior branch of AN.

In EMG studies (Table 3), compound muscle action potentials were recorded. For shoulder movement, nascent EMG potentials on pectoralis major (PM) muscles were detectable at a mean of $6.7 \pm 0.8$ months postoperatively (range 6-8 months), and on latissimus dorsi at $9.3 \pm 1.4$ months (range 8-12 months). All patients reported noticeable shoulder adduction at a mean of 10 months postoperatively (range 9-12 months). For elbow movement, nascent EMG potentials on triceps long head were detectable at $11.5 \pm 1.4$ months (range 10-14 months). All patients reported noticeable elbow extension at a mean of 14 months (range 12-17 months). For wrist and finger movement, nascent EMG potentials in the wrist extension muscles were detectable at 17.2 \pm 1.5 months (range 16-20 months) on extensor carpal radialis longus and extensor carpal radialis brevis in all patients, and $22.8 \pm 2.0$ months (range 20-26 months) for extensor digitorum and thumb extensors. Four were reported to have noticeable wrist extension and only 1 was reported with noticeable finger/thumb extension at the latest follow-up. In 3 patients, noticeable antagonistic movement of flexor carpi radialis (FCR) was recorded, and their nascent EMG potentials in FCR were detectable at $17.0 \pm 1.1$ months (range 16-19 months).

Regarding motor functional recovery assessment (Table 4), the average recovery at the latest follow-up was M2- for PM, M3 for latissimus dorsi, M3+ for triceps, M2 for wrist extensors, M1+ for FCR, and M1+ for digital extensors. All patients achieved a good or excellent result for shoulder adduction and elbow extension, with 5 of 6 achieving power of M3 or higher. The average shoulder abduction was $25^{\circ}-30^{\circ}$ in 3 patients, whereas $75^{\circ}-90^{\circ}$ was achieved in patients with the PN transfer. Elbow flexion was graded as excellent in 4 cases and good in 2 cases. Two patients obtained M3 for wrist extension, whereas the wrist flexion and finger extension were poor. Moreover, although there was long-lasting cocontraction of the C7 innervating muscles on both sides even 2 years postoperatively, independent movement on the injured side could still be strengthened, especially when firing muscles on the healthy side, which was estimated quantitatively as modified BMRC grade 1 .

For sensory recovery (Table 5), all patients achieved $\mathrm{S} 2$ in the index finger on the side of the injured upper limbs at the latest follow-up (except for one patient, who achieved only S1). The average 2-point discrimination (2PD) sensory function test result was $12 \mathrm{~mm}$ (range 10-15 $\mathrm{mm})$. For complications, no permanent impairment of donor limb function was reported. All patients experienced numbness and decreased 2-PD of the thumb and index and middle finger pulp; 5 of them recovered within 3 months and the other one within 6 months. Three patients experienced pain on the index finger, which all resolved within 3 months. One patient experienced a decrease of triceps muscle strength to $\mathrm{M} 3+$, which fully recovered within 1 month.

\section{Discussion}

\section{Anatomical Findings}

The CC7 is a potential donor nerve in treating brachial plexus injury ${ }^{6,11,28,34}$ and central neurological injuries..$^{14,37,39}$

TABLE 4. Motor functional recovery on affected side in 6 patients with TBPP

\begin{tabular}{ccccccc}
\hline Case No. & Pectoralis Major & Latissimus Dorsi & Triceps Brachii & Extensor Carpi & Flexor Carpi & Extensor Digitorum \& Pollicis \\
\hline 1 & M2+ & M3 & M4 & M2- & M2 & M1 \\
\hline 2 & M3 & M3 & M3+ & M2+ & M1 & M1 \\
\hline 3 & M1 & M3+ & M3+ & M1+ & M2- & M1 \\
\hline 4 & M3 & M3 & M4 & M1+ & M2 & M1 \\
\hline 5 & M1 & M3+ & M2 & M3+ & M1 & M2+ \\
\hline 6 & M1 & M4 & M4- & M3- & M1 & M1+ \\
\hline
\end{tabular}

The modified BMRC scale was used for scoring. 
TABLE 5. Sensory function on both sides in 6 patients with TBPP

\begin{tabular}{|c|c|c|c|c|c|}
\hline \multirow[b]{2}{*}{$\begin{array}{c}\text { Case } \\
\text { No. }\end{array}$} & \multicolumn{3}{|c|}{ Affected Side } & \multicolumn{2}{|c|}{ Unaffected Side } \\
\hline & $\begin{array}{l}\text { Index } \\
\text { Finger }\end{array}$ & $\begin{array}{l}2-P D \\
(\mathrm{~mm})\end{array}$ & $\begin{array}{l}\text { Paresthesia } \\
\text { When Touching } \\
\text { Unaffected } \\
\text { Index Finger }\end{array}$ & $\begin{array}{l}\text { Duration of } \\
\text { Numbness } \\
\text { (mos) }\end{array}$ & $\begin{array}{l}\text { Duration of } \\
\text { Unbearable } \\
\text { Pain (mos) }\end{array}$ \\
\hline 1 & S2 & 11 & Yes & 3 & NA \\
\hline 2 & S2 & 11 & Yes & 3 & 1 \\
\hline 3 & S1 & 15 & Yes & 6 & 3 \\
\hline 4 & S2 & 10 & Yes & 3 & 1 \\
\hline 5 & S2 & 12 & Yes & 3 & NA \\
\hline 6 & S2 & 11 & Yes & 3 & NA \\
\hline
\end{tabular}

$\mathrm{NA}=$ not applicable

The C7 nerve root possesses $25,000-40,000$ fibers, ${ }^{16}$ providing a great source of nerve fibers, ${ }^{4,10,11,23}$ Current understanding of the brachial plexus ${ }^{1,12,19,25}$ would suggest that C7 myotome contributes to the movements of different muscles, and there does not appear to be an action that is controlled solely by C7. Hence there is the potential that the nerve root could be expendable, which is in accordance with the findings in our functional neuroanatomy study for C7 nerve distribution-that LPN, TN, LTN, and LHTB were all supplied mainly by the components of C7 nerve root. The anterior division of the $\mathrm{C} 7$ nerve makes its contribution in the median and musculocutaneous nerves, whereas the posterior division of the $\mathrm{C} 7$ root participates in the $\mathrm{AN}, \mathrm{TN}$, and radial nerves.

Therefore, the motor fibers of the $\mathrm{C} 7$ nerve root are dispersive. The $\mathrm{C} 7$ root is presumed to functionally innervate muscles of multiple joints such as shoulder (latissimus dorsi, PM); elbow (triceps); wrist (extensor carpi radialis); and hand (extensor digitorum) - the functional restoration of these joints is observed primarily in our clinical study. In our anatomy study, the pattern of C7 nerve root branching was predictable. The cadaver study results were in accordance with previous research overall, except for particular findings. For instance, a specimen study of 100 adult cadavers showed that the LPN mostly originated from the anterior division of the upper trunk proximal to the point of lateral cord formation (88 of 100 cases). ${ }^{1}$ However, in our study the LPN mostly arose from the anterior divisions of the upper and middle trunk (8 in 10 sides) or as a single root from the lateral cord ( 2 in 10 sides), across the axillary artery and vein, and entering the clavicular part of the PM.

The distance measured from the $\mathrm{C} 7$ nerve root to the nerve entry point of the PM was $10.3 \pm 1.38 \mathrm{~cm}$ (range $7.9-12.0 \mathrm{~cm}$ ). Moreover, in the present study we found that the TN arose from the posterior division of the middle and lower trunk, in which the branch from the middle trunk became thicker in diameter under loupe magnification $\times 2.5$. The distance from the $\mathrm{C} 7$ root to the $\mathrm{TN}$ entry point of the muscle was $21.7 \pm 1.77 \mathrm{~cm}$ (range $18.5-25.0 \mathrm{~cm}$ ). $\mathrm{Lu}$ et al..$^{18}$ published their prediction of the origin of the dominant nerve of latissimus dorsi muscle, which they determined by intraoperative electrophysiological examination. They put forth a similar conclusion that the amplitude ratios suggested that the middle trunk exerted the greatest influence over the latissimus dorsi muscle $(41.3 \% \pm 2.56 \%)$, and their conclusion has been further supported by histochemical staining, which showed that approximately $52 \%$ of motor fibers in the TN came from C7.19,35

Regardless of research techniques, Lu et al. also proposed that TN fibers originate from each of the three trunks, whereas in our study TN arose from the middle trunk mainly and also the lower trunk. The anatomy of the LTN has not been fully elucidated. In our study it was found that the LTN was formed by branches arising from the $\mathrm{C} 5, \mathrm{C} 6$, and $\mathrm{C} 7$ nerve roots, and that the distance between the $\mathrm{C} 7$ nerve root and the LTN entry of the muscle was $24.1 \pm 3.26 \mathrm{~cm}$ (range $20.5-30.0 \mathrm{~cm}$ ). In the present study, the $\mathrm{C} 5$ and $\mathrm{C} 6$ branches joined beneath the scalenus muscles to form the upper division, and the branch from C7 contributed to the lower part of LTN, whereas Hamada et al. ${ }^{12}$ found that the upper part was supplied mainly by the $\mathrm{C} 5$ nerve root, and the $\mathrm{C} 4, \mathrm{C} 6$, or $\mathrm{C} 7$ nerve roots also had multiple branches in 64 of 70 dissections. For the LHTB, it came from the middle and lower trunk. The distance from the $\mathrm{C} 7$ root to the nerve entry point of triceps muscle was $23.4 \pm 1.82 \mathrm{~cm}$ (range 19.5-26.0 cm).

The different lengths of the nerve branches, which indicate the distance and time of nerve reinnervation, were particularly recorded in this study (Table 2). The time and order of recovery in specific target muscles could be estimated given the routes of the nerve transfer. Because the LPN had the shortest distance from the nerve root to nerve entry point of the clavicular part of the PM, it is presumed to be the first reinnervated muscle, which would be further supported by first emergence of the Tinel sign and EMG recording signals clinically. In other words, the PM or LPN function can be used as the first observation and assessment after the CC7 surgery to determine nerve reinnervation, to assess the effect of the surgery, and to achieve early intervention and rehabilitation.

From the cadaver study, the fact that there were consistent degradations of components from the $\mathrm{C} 7$ nerve root to the LPN, TN, LTN, and LHTB (Table 2, Fig. 2) would be solid evidence to support the hypothesis that a comprehensive functional recovery of the upper limb could be achieved on successful C7 regeneration. TBPP results in the most catastrophic prognosis, ${ }^{3}$ and current concepts for treatment are primarily focused on neurotization. Therefore, based on anatomical studies, we designed the procedure of $\mathrm{CC} 7$ transfer to repair the avulsed $\mathrm{C} 7$ nerve root, and we combined electrophysiological and functional measures to explore the utility of $\mathrm{C} 7$ nerve as a recipient for TBPP. The CC7 transfer route is another important factor. Here, the feasibility of the retrosternocleidomastoid muscle route with cabled sural nerve grafts was tried and applied, because one of the major aims of the $\mathrm{CC} 7$ transfer deals with providing a route that is both short and safe to the maximum extent. As a matter of fact, several routes for CC7 transfer have been stated. Mcguiness and Kay ${ }^{21}$ (2002) transferred the nerve through the retropharyngeal space. Xu et al..$^{36}(2008)$ accomplished the transfer through a tunnel posterior to the scalenus anterior muscle. Chuang and Hernon ${ }^{6}$ (2012) performed a subcutaneous transfer for monitoring the progression of Tinel sign, although the 
nerve route was longer than the prevertebral route. Similarly, Zhang and $\mathrm{Gu}^{38}$ (2011) used a cross-chest subcutaneous tunnel. In the current investigation, based on the anatomical study, the retrosternocleidomastoid approach was used with the average nerve graft of $9.5 \mathrm{~cm}$, which made a tension-free suture and a relatively short graft distance as compared to subcutaneous tunnel, and it was easy to perform the procedure and follow the Tinel sign compared with the prespinal route.

\section{Clinical Series}

As a result of the anatomical findings, comprehensive functional improvements were achieved on the injured upper limbs, especially for shoulder adduction and elbow extension functions. These functions-with a grade of M3 or greater attained by 5 of 6 patients-implied that the C7 nerve root commanded a stable and dominant role over the latissimus dorsi, PM, and triceps brachii. It showcased consistency with our anatomical study wherein the muscle branches of the $\mathrm{C} 7$ components were confirmed. Besides, according to the EMG study the PM that was dominated by LPN had an earlier recovery. Soon after, successful reinnervation of latissimus dorsi guaranteed a stronger shoulder adduction. This recovery time and the order of restoration of specific muscles could also be estimated by the distances measured from the $\mathrm{C} 7$ nerve root to the nerve entry point of related muscles. Regarding motor recovery, our results seem superior to previous researches. For instance, Gao et al. ${ }^{8}$ discovered that the effective recovery rate, which was classified as a muscle grade of M3 or higher, was $56 \%$ in the study of ICN transfer to the nerve of the triceps long head in TBPP. Arnal et al. ${ }^{2}$ included 21 patients with TBPP in their study, in which they grafted C5 root on the nerve of the triceps long head and, at a mean follow-up of 22 months, $62 \%$ of their patients had achieved M3 or higher.

Besides shoulder adduction, shoulder abduction and external rotation also play an important role for a comprehensive shoulder function, for which the muscles were innervated by SSN and AN. Various reconstructive procedures have been developed. Available donors include the SAN, ICN, PN, proximal C5-6 stump, CC7, and the triceps branch of the radial nerve. Sammer et al. ${ }^{24}$ proposed hemiCC7 as a neurotizer for shoulder function, and attained only $23 \%$ acceptable shoulder abduction. Chuang and colleagues $^{5,7}$ reported 8 different combinations of neurotization procedures for shoulder reconstruction. They concluded that simultaneous neurotization to the SSN and AN obtained better results than just neurotization to the SSN. Tu et al. ${ }^{31}$ also achieved a similar result by dual neurotization procedures (SAN-SSN combined with ICN-AN). In our case series dual neurotization procedures were applied (SAN-SSN combined with PN-AN). With this procedure significant shoulder abduction was achieved, together with strong shoulder adduction, which provided sound shoulder function.

The recovery of wrist and finger extension did not satisfy our expectations, even though the compound muscle action potentials had been recorded at $17.2 \pm 1.5$ months postoperatively on wrist extensors and at $22.8 \pm 2.0$ months on digital extensors. Moreover, finger extension recovered with more difficulty than the wrist; the latest follow-up (average 30 months postoperatively) showed that just 2 patients achieved a grade of M3 for wrist extension, whereas only 1 received a grade of $\mathrm{M} 2$ for finger extension. Although the longest follow-up reached 40 months, patients' functions had not changed in the last 10 months. This demonstrates that the recovery of wrist and digital extension is not reliable and stable. The following reasons are likely to explain. 1) These patients mostly achieved a much better improvement in shoulder and arm function postoperatively (Table 4). It implies that the total number of fibers from the C7 nerve root to the shoulder and elbow is greater than that to the wrist and fingers. Experiments with different donorto-target axon ratios in rabbits have demonstrated helpful motor recovery starting at a 1:3 ratio. ${ }^{20}$ Research in rats also revealed that at least $30 \%$ of the original motor neurons were needed to achieve normal muscle strength. ${ }^{30}$ In the present study, the difference in the number of fibers led to the different recoveries in target muscles, and it could predict that better recovery was unlikely to be achieved even in longer follow-up. 2) Persistent strong wrist flexion antagonism may be another reason for the unsatisfactory wrist recovery. Anatomy and EMG studies have shown that FCR also shared a proportion of fibers from the $\mathrm{C} 7$ nerve root, thus causing ineffective wrist movement after regeneration, because there was antagonism of wrist extension and flexion. For these patients with Tinel sign at the supinator tunnel, the neurolysis for the deep branch of the radial nerve was done by opening the supinator tunnel; however, it was found that the finger extension recovered but the wrist did not, which further implied that the antagonism of wrist flexion led to the poor recovery of wrist movement.

Cocontraction movement is proposed to be another interesting phenomenon-patients could obtain more improvement in shoulder and elbow functions by simultaneous firing of shoulder adduction or elbow extension on the healthy side, which was especially so with the same movement on the injured side. This phenomenon may be attributed to the fact that the CC7 nerve delivers the nerve fibers connecting both sides, and in this procedure the reshaping of the two hemispheres is easier. ${ }^{13,15,27}$ With regard to the similarity of diameters and fibers between CC7 and avulsed C7 nerve roots, neural connection was easily established, which was favorable for the functional recovery of the limb ${ }^{22}$ and brain plasticity. ${ }^{39}$

There are some limitations in this study; it lacks high statistical power on account of the small number of samples. Because 2 cases were excluded from the initial 8 patients in this study, which might affect the results, special cautions should be taken for patients with severe concomitant soft-tissue damage, and more cases are needed for further research. Moreover, it was apparent in the anatomical study that the LTN got proportional fibers from the C7 nerve root. However, it was not practical for us to study the serratus reinnervation because all the nerve roots were avulsed, making it infeasible to repair the LTN at the C7 nerve root level.

\section{Conclusions}

The C7 nerve root contributes consistently to the LPN, 
the TN, and the LHTB of the radial nerve. CC7 to $\mathrm{C} 7$ nerve transfer is a reconstructive option in the overall management plan for TBPP. It was safe and effective in restoring shoulder adduction and elbow extension in this patient series. However, recoveries of wrist and finger extensions were poor.

\section{Acknowledgments}

This work was supported by Priority Among Priorities of Shanghai Municipal Clinical Medicine Center (2017ZZ01006 [Wen-Dong $\mathrm{Xu}$ ]); the National Funds for Distinguished Young Scientists (81525009 [Wen-Dong Xu]); the National Natural Science Foundation of China (81171151 [Wen-Dong Xu], 81501945 [Su Jiang], 81371389 [Fei Ding], and 31500927 [Qian-Ru He]); National Key R\&D Program of China (2017YFC0840100 and 2017YFC0840106 [Wen-Dong Xu]); and Key Clinical Program of the Ministry of Health (2010-439 Ministry of Health [Shu-Sen Cui]).

\section{References}

1. Arad E, Li Z, Sitzman TJ, Agur AM, Clarke HM: Anatomic sites of origin of the suprascapular and lateral pectoral nerves within the brachial plexus. Plast Reconstr Surg 133:20e$27 \mathrm{e}, 2014$

2. Arnal M, Cambon A, Marcheix PS, Belkhayar Z: Restoration of elbow and hand function in total brachial plexus palsy with intercostal nerves and C5 root neurotization. Results in 21 patients. Hand Surg Rehabil 35:283-287, 2016

3. Bonnard C, Anastakis D: Complete palsy, in Gilbert A (ed): Brachial Plexus Injuries. London: Martin Dunitz, 2001, pp 67-75

4. Bonnel F: Microscopic anatomy of the adult human brachial plexus: an anatomical and histological basis for microsurgery. Microsurgery 5:107-118, 1984

5. Chuang DC: Nerve transfers in adult brachial plexus injuries: my methods. Hand Clin 21:71-82, 2005

6. Chuang DC, Hernon C: Minimum 4-year follow-up on contralateral C7 nerve transfers for brachial plexus injuries. J Hand Surg Am 37:270-276, 2012

7. Chuang DC, Lee GW, Hashem F, Wei FC: Restoration of shoulder abduction by nerve transfer in avulsed brachial plexus injury: evaluation of 99 patients with various nerve transfers. Plast Reconstr Surg 96:122-128, 1995

8. Gao K, Lao J, Zhao X, Gu Y: Outcome after transfer of intercostal nerves to the nerve of triceps long head in 25 adult patients with total brachial plexus root avulsion injury. $\mathbf{J}$ Neurosurg 118:606-610, 2013

9. Gu Y: Functional motor innervation of brachial plexus roots. An intraoperative electrophysiological study. Chin Med J (Engl) 109:749-751, 1996

10. Gu YD, Chen DS, Zhang GM, Cheng XM, Xu JG, Zhang LY, et al: Long-term functional results of contralateral C7 transfer. J Reconstr Microsurg 14:57-59, 1998

11. Gu YD, Zhang GM, Chen DS, Yan JG, Cheng XM, Chen L: Seventh cervical nerve root transfer from the contralateral healthy side for treatment of brachial plexus root avulsion. J Hand Surg Br 17:518-521, 1992

12. Hamada J, Igarashi E, Akita K, Mochizuki T: A cadaveric study of the serratus anterior muscle and the long thoracic nerve. J Shoulder Elbow Surg 17:790-794, 2008

13. Hua XY, Liu B, Qiu YQ, Tang WJ, Xu WD, Liu HQ, et al: Long-term ongoing cortical remodeling after contralateral C-7 nerve transfer. J Neurosurg 118:725-729, 2013

14. Hua XY, Qiu YQ, Li T, Zheng MX, Shen YD, Jiang S, et al: Contralateral peripheral neurotization for hemiplegic upper extremity after central neurologic injury. Neurosurgery 76:187-195, 2015
15. Jiang S, Li ZY, Hua XY, Xu WD, Xu JG, Gu YD: Reorganization in motor cortex after brachial plexus avulsion injury and repair with the contralateral $\mathrm{C} 7$ root transfer in rats. Microsurgery 30:314-320, 2010

16. Liu Y, Zhou X, Ma J, Ge Y, Cao X: The diameters and number of nerve fibers in spinal nerve roots. J Spinal Cord Med 38:532-537, 2015

17. Lu W, Xiao J, Xu J, He Q, Li J, Wang D, et al: [Study on the quantity and distribution of motor fiber of rat's C7 nerve root.] Zhongguo Xiu Fu Chong Jian Wai Ke Za Zhi 19:857-859, 2005 (Chinese)

18. $\mathrm{Lu} \mathrm{W}, \mathrm{Xu}$ J, Wang D: Intraoperative decision making by electrophysiological detection of the origin of thoracodorsal nerve in the $\mathrm{C} 7$ nerve root transfer procedure. J Reconstr Microsurg 27:1-4, 2011

19. Lu W, Xu JG, Wang DP, Gu YD: Microanatomical study on the functional origin and direction of the thoracodorsal nerve from the trunks of brachial plexus. Clin Anat 21:509-513, 2008

20. Lutz BS, Chuang DC, Chuang SS, Hsu JC, Ma SF, Wei FC: Nerve transfer to the median nerve using parts of the ulnar and radial nerves in the rabbit-effects on motor recovery of the median nerve and donor nerve morbidity. J Hand Surg Br 25:329-335, 2000

21. Mcguiness CN, Kay SP: The prespinal route in contralateral C7 nerve root transfer for brachial plexus avulsion injuries. J Hand Surg Br 27:159-160, 2002

22. Millesi H: Bridging defects: autologous nerve grafts. Acta Neurochir Suppl 100:37-38, 2007

23. Narakas A: Surgical treatment of traction injuries of the brachial plexus. Clin Orthop Relat Res (133):71-90, 1978

24. Sammer DM, Kircher MF, Bishop AT, Spinner RJ, Shin AY: Hemi-contralateral C7 transfer in traumatic brachial plexus injuries: outcomes and complications. J Bone Joint Surg Am 94:131-137, 2012

25. Sinha S, Prasad GL, Lalwani S: A cadaveric microanatomical study of the fascicular topography of the brachial plexus. J Neurosurg 125:355-362, 2016

26. Songcharoen P, Wongtrakul S, Mahaisavariya B, Spinner RJ: Hemi-contralateral C7 transfer to median nerve in the treatment of root avulsion brachial plexus injury. J Hand Surg Am 26:1058-1064, 2001

27. Sun G, Wu Z, Wang X, Tan X, Gu Y: Nerve transfer helps repair brachial plexus injury by increasing cerebral cortical plasticity. Neural Regen Res 9:2111-2114, 2014

28. Terzis JK, Kokkalis ZT: Selective contralateral C7 transfer in posttraumatic brachial plexus injuries: a report of 56 cases. Plast Reconstr Surg 123:927-938, 2009

29. Terzis JK, Kostopoulos VK: Free muscle transfer in posttraumatic plexopathies: part III. The hand. Plast Reconstr Surg 124:1225-1236, 2009

30. Tötösy de Zepetnek JE, Zung HV, Erdebil S, Gordon T: Innervation ratio is an important determinant of force in normal and reinnervated rat tibialis anterior muscles. J Neurophysiol 67:1385-1403, 1992

31. Tu YK, Tsai YJ, Chang CH, Su FC, Hsiao CK, Tan JS: Surgical treatment for total root avulsion type brachial plexus injuries by neurotization: a prospective comparison study between total and hemicontralateral $\mathrm{C} 7$ nerve root transfer. Microsurgery 34:91-101, 2014

32. Vanaclocha V, Herrera JM, Verdu-Lopez F, Gozalbes L, Sanchez-Pardo M, Rivera M, et al: Transdiscal C6-C7 contralateral C7 nerve root transfer in the surgical repair of brachial plexus avulsion injuries. Acta Neurochir (Wien) 157:2161-2167, 2015

33. Waikakul S, Orapin S, Vanadurongwan V: Clinical results of contralateral C7 root neurotization to the median nerve in brachial plexus injuries with total root avulsions. J Hand Surg Br 24:556-560, 1999 
34. Wang SF, Li PC, Xue YH, Yiu HW, Li YC, Wang HH: Contralateral C7 nerve transfer with direct coaptation to restore lower trunk function after traumatic brachial plexus avulsion. J Bone Joint Surg Am 95:821-827, S1-S2, 2013

35. Xu JG, Hu SN, Wang H, Gu YD: Histochemical study on C7 roots and its clinical significance. Chin J Clin Anat 14:243245, 1996

36. Xu L, Gu Y, Xu J, Lin S, Chen L, Lu J: Contralateral C7 transfer via the prespinal and retropharyngeal route to repair brachial plexus root avulsion: a preliminary report. Neurosurgery 63:553-559, 2008

37. Xu WD, Hua XY, Zheng MX, Xu JG, Gu YD: Contralateral C7 nerve root transfer in treatment of cerebral palsy in a child: case report. Microsurgery 31:404-408, 2011

38. Zhang CG, Gu YD: Contralateral C7 nerve transfer-our experiences over past 25 years. J Brachial Plex Peripher Nerve Inj 6:10, 2011

39. Zheng MX, Hua XY, Feng JT, Li T, Lu YC, Shen YD, et al: Trial of contralateral seventh cervical nerve transfer for spastic arm paralysis. N Engl J Med 378:22-34, 2018

\section{Disclosures}

The authors report no conflict of interest concerning the materials or methods used in this study or the findings specified in this paper.

\section{Author Contributions}

Conception and design: WD Xu, GB Wang, Yu, Gu, JG Xu, Jiang. Acquisition of data: Yu, XM Wang, Feng, Li, Chen, He, Ding, Cui. Analysis and interpretation of data: GB Wang, Jiang. Drafting the article: WD Xu, GB Wang, Yu, Jiang. Critically revising the article: Ng, Lei, He, Ding, Cui, Gu, JG Xu. Reviewed submitted version of manuscript: Ng. Statistical analysis: Yu, Lei, Qiu. Administrative/technical/material support: Ng, Qiu. Study supervision: WD Xu, Jiang. Anatomy study: WD Xu, GB Wang, Qiu.

\section{Correspondence}

Wen-Dong Xu: Huashan Hospital, Fudan University, Shanghai, China. wendongxu@fudan.edu.cn. 